\title{
Inhibition is picky: Shape difference is a necessary condition for attentional inhibition of irrelevant objects
}

\author{
Peter WÜHR \\ Friedrich-Alexander-Universität Erlangen-Nürnberg, Erlangen, Germany \\ AND \\ Christian Frings \\ Universität des Saarlandes, Saarbrücken, Germany
}

\begin{abstract}
The present study investigated the conditions for attentional amplification in the processing of relevant objects and for attentional inhibition in the processing of irrelevant objects. Participants reported the color of one of two superimposed objects that was specified by occlusion. Irrelevant color words were presented as part of the relevant object, as part of the irrelevant object, or in the background; the words were either congruent or incongruent with the color of the relevant object. The size of the congruency effects provided a measure of the (relative) strength of the processing of the objects and the background. Finally, the two objects had the same shape in one session and different shapes in another session. In both sessions, results showed larger Stroop effects from words belonging to the relevant object than from words in the background, indicating attentional amplification of the relevant object that was unaffected by object similarity. In contrast, smaller Stroop effects from words belonging to the irrelevant object than from words in the background occurred only when the two objects differed in shape. The latter result suggests that a shape difference is both a necessary and a sufficient condition for inhibiting the processing of an irrelevant visual object.
\end{abstract}

In order to facilitate the processing of action-related information, humans can selectively attend to relevant visual objects at the expense of irrelevant objects (see, e.g., Duncan, 1984). The mechanisms of object-based attentional selection are still a matter of considerable debate, however: Does object-based attention encompass only a single mechanism that selectively amplifies the processing of relevant objects (Kahneman \& Henik, 1981)? Or does object-based attention engage two mechanisms: one that amplifies the processing of the relevant object, and another that suppresses the processing of irrelevant objects (Houghton \& Tipper, 1994; Keele \& Neill, 1978)? The present article reports converging evidence for the latter view. In addition, the present article presents the first evidence for the notion that attentional amplification and inhibition may require different preconditions.

The primary means for studying the mechanisms of object-based attention has been the negative-priming (NP) paradigm. In this paradigm, participants are required to respond to consecutive pairs of displays (i.e., a prime and a probe display) that contain both a relevant and an irrelevant object. For example, Tipper (1985) presented superimposed drawings of two objects in different colors; participants named the red object and ignored the green object.
Tipper manipulated the relationship between the objects in the prime and probe displays. In the control condition, the prime and the probe displays contained different objects. In the attended-repetition condition, the same object was presented as the prime target and as the probe target, but the distractor object changed. Finally, in the ignored-repetition condition, the prime distractor was also presented as the probe target together with a new distractor object. Response times (RTs) were faster in the attended-repetition condition than in the neutral conditions, reflecting positive priming, whereas RTs were slower in the ignored-repetition condition, reflecting negative priming. This pattern of results has been replicated in a large number of studies, using a variety of stimuli, tasks, and participant populations (for reviews, see Fox, 1995; Tipper, 2001).

Several authors have interpreted the pattern of priming effects that have been observed with superimposed objects as evidence for dual mechanisms of object-based attentional selection (e.g., Frings \& Wühr, 2007; Houghton \& Tipper, 1994; Tipper, 1985, 2001). According to this view, selection between target and distractor objects in the prime display involves amplification of the target object and inhibition of the distractor object. Amplification of prime targets facilitates subsequent processing of probe

P.Wühr, wuehr@fk14.tu-dortmund.de 
targets in the attended-repetition condition; inhibition of prime distractors interferes with subsequent processing of probe targets in the ignored-repetition condition. The sequential nature of the NP paradigm, however, produces problems for explaining priming effects as the aftereffects of attentional selection during prime-display processing. First, the pattern of priming effects is open to alternative, nonattentional explanations (Milliken, Joordens, Merikle, \& Seiffert, 1998; Neill \& Mathis, 1998; Rothermund, Wentura, \& De Houwer, 2005). Second, the sequential nature of the paradigm makes it impossible to determine whether both amplification and inhibition are in fact involved in selecting the prime target object. For example, inhibition could also be applied to the distractor representation after selection has been achieved through amplification of the target representation alone.

Wühr and colleagues have developed a new experimental paradigm for investigating object-based attentional selection that does not face the problems of the NP paradigm. In the first series of studies, Wühr and colleagues presented two superimposed rectangles to the participants (see, e.g., Wühr \& Waszak, 2003). Each object and the background appeared in a different color. Participants reported the color of the occluding rectangle (the relevant object) and ignored the occluded rectangle (the irrelevant object). Two identical color words were presented in the relevant object, in the irrelevant object, or in the background. The color words were either congruent or incongruent with the color of the relevant object, enabling the measurement of Stroop-like congruency effects. The size of the congruency effects reflected the strength of the processing of the object or area to which the words belonged. In particular, if object-based attention amplified the processing of the relevant object, then words that were in the relevant object should have produced larger Stroop effects than did words that were in the background. Conversely, if object-based attention inhibited the processing of irrelevant objects, then words that were in the irrelevant object should have produced smaller Stroop effects than did words that were in the background.

Using displays with crossed rectangles, Wühr and colleagues consistently found that words that were in the relevant object produced larger Stroop effects than did words that were in the irrelevant object or words that were in the background, whereas Stroop effects did not differ in the two latter conditions (Wühr, 2007; Wühr \& Waszak, 2003; Wühr $\&$ Weltle, 2005). These results suggest that object-based attention amplifies the processing of the relevant object but does not inhibit the processing of the irrelevant object.

In a more recent study, Wühr and Frings (2008) reported evidence suggesting that humans can also inhibit the processing of an irrelevant object. In this study, Wühr and Frings presented two overlapping objects that differed in their shape (e.g., a circle and a square), with the relative locations of the two objects varied randomly from trial to trial. Participants reported the color of the relevant object and ignored the irrelevant object. Again, congruent or incongruent color words were presented as part of the relevant object, as part of the irrelevant object, or in the background. In contrast to previous studies, Wühr and Frings observed both larger Stroop effects from words that were in the relevant object and smaller Stroop effects from words that were in the irrelevant object when compared with Stroop effects from words that were in the background. The latter result suggests that participants can in fact inhibit the processing of an irrelevant visual object, but only if the irrelevant object differs in shape from the relevant object. Several methodological differences between the two types of studies, however, make it difficult to pinpoint the exact conditions for attentional inhibition of irrelevant objects.

The purpose of the present study was to investigate whether a shape difference between two objects is sufficient to inhibit the processing of the irrelevant object. Participants were presented with displays containing two superimposed objects (see Figure 1 for examples). To measure the effects of object-based attention, we presented congruent or incongruent color words as part of the relevant object (Figures 1A and 1B), as part of the irrelevant object (Figures $1 \mathrm{C}$ and $1 \mathrm{D}$ ), or in the background (Figures 1E and 1F). The critical manipulation concerned the shape of the irrelevant object. As was the case in the study by Wühr and Waszak (2003), the irrelevant object had the same shape as the relevant object in one session (e.g., Figure 1A). In the other session, the shape of the irrelevant object was different from that of the relevant object (e.g., Figure 1B), as was the case in the study by Wühr and Frings (2008). We expected Stroop effects from words in the relevant object to be larger than those from words in the background, reflecting attentional amplification of the relevant object, in both sessions. By contrast, we expected Stroop effects from words that were in the irrelevant object to be smaller than those from words that were in the background when the shapes differed, but not when the shapes were the same. The latter result would provide the first direct evidence that a shape difference between a relevant and an irrelevant object provides both a necessary and a sufficient condition for inhibiting the processing of the irrelevant visual object.

\section{METHOD}

\section{Participants}

Thirty-two volunteers ( 17 female, 15 male) with a mean age of 24 years participated in two sessions on separate days. Each session lasted about $30 \mathrm{~min}$, and participants received $€ 10$ for attendance. All participants were native German speakers with normal (or corrected-to-normal) visual acuity and with normal color vision.

\section{Apparatus and Stimuli}

The experiment took place in a dimly lit room. The participants sat in front of a 17-in. color monitor. A head- and chinrest constrained viewing distance to $50 \mathrm{~cm}$. Participants responded by speaking into a microphone, which triggered a voice key measuring RTs to the millisecond. A personal computer controlled the presentation of stimuli and collected vocal RTs. The fixation cross was a plus sign $(4 \times 4 \mathrm{~mm})$. Each stimulus display consisted of two superimposed objects forming a cross, presented at screen center. Each object was either a rectangle or an ellipse with a length of $80 \mathrm{~mm}$ and a width of $20 \mathrm{~mm}$ (see Figure 1 for examples). Participants had to name the color of one object (i.e., the relevant object) and ignore the color of the other object (i.e., the irrelevant object).

In each stimulus display, the background, the irrelevant object, and the relevant object appeared in different colors. The four colors 


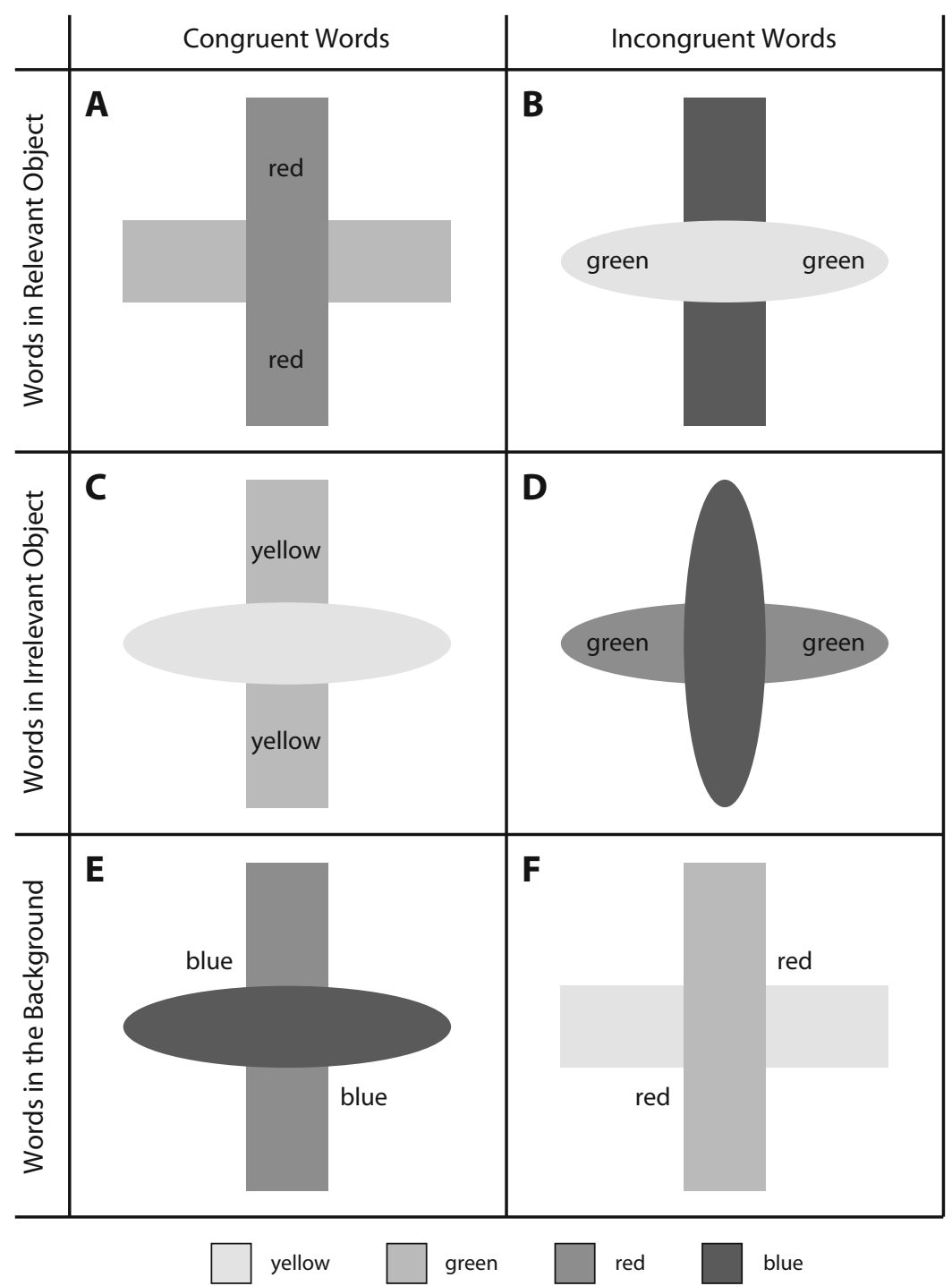

Figure 1. Sample displays that were used in the present experiment. Panels A-F show examples for the six experimental conditions resulting from the presentation of congruent or incongruent words in the relevant object, the irrelevant object, or the background. Note that the relevant object is always the occluding object. The displays in panels $A, D$, and $F$ show two objects of the same shape (i.e., either two rectangles or two ellipses in different orientations), whereas the displays in panels $B, C$, and $E$ show two objects with different shapes (i.e., a rectangle and an ellipse).

used were blue, green, red, and yellow. In order to provoke Stroop effects, two identical color words appeared in one of the objects or in the background. Color words were the German words for blue, green, red, and yellow, subtending between $8 \times 4 \mathrm{~mm}$ and $15 \times 6 \mathrm{~mm}$. The spatial distance between the fixation point and the center of a word was $25 \mathrm{~mm}$ in all conditions. Words that were inside the objects occurred on the horizontal or vertical meridian, whereas words that were outside the objects occurred on a diagonal.

\section{Procedure}

At the beginning of each session, the instructions appeared on the screen, describing the displays and the task. Half of the participants were instructed to report the color of the occluding object (regardless of shape); the other half were instructed to report the color of the occluded object (regardless of shape). In addition, participants were told to ignore the words that were presented in the displays.
Finally, instructions encouraged participants to fixate the plus sign, respond loudly, and respond as quickly and accurately as possible. The instructions were followed by one block of 20 practice trials.

Both the selection criterion (i.e., the depth position of the relevant object) and the shape of the relevant object (rectangle or ellipse) remained constant across both sessions. The only difference between sessions concerned the similarity between the relevant and the irrelevant objects. Specifically, the irrelevant object had the same shape as the relevant object in one session (i.e., two rectangles or two ellipses), whereas the irrelevant object had a different shape than the relevant object in the other session (i.e., one rectangle and one ellipse). The order of these two conditions was counterbalanced across participants.

The 384 experimental trials were divided into 18 blocks of 20 trials and 1 block of 24 trials. At the beginning of each block, the string "Press a key" appeared. The keypress started a block of trials, each 
Table 1

Mean Response Times (RTs, in Milliseconds) and Percentages of Errors in the Present Experiment As a Function of Object Similarity, Word Location, and Congruency

\begin{tabular}{|c|c|c|c|c|c|c|c|c|c|c|c|c|}
\hline & \multicolumn{6}{|c|}{ Similar Objects } & \multicolumn{6}{|c|}{ Dissimilar Objects } \\
\hline & \multicolumn{2}{|c|}{ Relevant Object } & \multicolumn{2}{|c|}{ Irrelevant Object } & \multicolumn{2}{|c|}{ Background } & \multicolumn{2}{|c|}{ Relevant Object } & \multicolumn{2}{|c|}{ Irrelevant Object } & \multicolumn{2}{|c|}{ Background } \\
\hline & RT & $\%$ Errors & RT & $\%$ Errors & RT & $\%$ Errors & RT & $\%$ Errors & RT & $\%$ Errors & RT & $\%$ Errors \\
\hline Congruent & 606 & 0.1 & 599 & 0.7 & 604 & 0.6 & 593 & 0.1 & 601 & 0.1 & 594 & 0.1 \\
\hline Incongruent & 724 & 11.1 & 647 & 3.5 & 654 & 4.9 & 706 & 10.7 & 643 & 3.8 & 656 & 5.0 \\
\hline Stroop effect & 118 & 11.0 & 48 & 2.8 & 50 & 4.3 & 113 & 10.6 & 42 & 3.7 & 62 & 4.9 \\
\hline
\end{tabular}

Note-Stroop effect $=$ incongruent - congruent condition .

of which contained the following events: First, the fixation cross appeared on the screen for $500 \mathrm{msec}$ on a gray background; then, after a blank of $100 \mathrm{msec}$, the stimulus display was presented for $400 \mathrm{msec}$; an empty gray screen followed the stimulus display and remained onscreen for $1,000 \mathrm{msec}$; after the last trial in a block, the string "Press a key" appeared, and the participant could take a rest.

The participants' performance was monitored online by the experimenter, who sat outside the experimental chamber. The experimenter heard the participants' responses via earphones and compared them with the correct answers that were shown on a second monitor. Each error was recorded in a list.

\section{Design}

The experiment used a $2 \times 3 \times 2$ within-subjects design. The first factor, object similarity, was manipulated between sessions. The order of conditions was counterbalanced across participants. The second factor was word location. The two color words were presented as parts of the relevant object, as parts of the irrelevant object, or in the background (see Figure 1 for examples). The third factor was congruency. The irrelevant words were either congruent or incongruent with respect to the correct color-naming response (i.e., the color of the relevant object). Note that the factorial combination of the four colors (of the relevant object) and the four color words revealed $25 \%$ congruent and $75 \%$ incongruent conditions. The color words were always incongruent with the color of the irrelevant object and with that of the background. Stroop effects were computed as the difference between congruent and incongruent trials.

\section{RESULTS}

\section{Response Times}

The results from 1 participant were not analyzed further, because his error rate $(M=14 \%)$ exceeded the error rate of the whole sample $(M=3.5 \%, S D=1.9 \%)$ by more than three standard deviations; thus, results from 31 participants were analyzed further. All trials containing RTs below $150 \mathrm{msec}(<1.0 \%)$ or above $1,500 \mathrm{msec}(<1.0 \%)$ were also dropped from further analysis. The mean RT values and error percentages are presented in Table 1. Preliminary analyses showed that neither the order of similarity conditions nor the location of the relevant object (occluding vs. occluded object) had significant effects; the results of these two factors are therefore not reported.

Mean RTs were entered into a 2 (object similarity) $\times$ 3 (word location) $\times 2$ (congruency) MANOVA using Pillai's trace as the criterion. The main effect of object similarity was not significant $\left[F(1,30)=1.09, p=.31, \eta_{\mathrm{p}}^{2}=\right.$ $.04]$. A significant main effect of word location $[F(2,29)=$ 74.10, $\left.p<.001, \eta_{\mathrm{p}}^{2}=.84\right]$ indicated longer RTs when words appeared in the relevant object $(657 \mathrm{msec})$ than when words appeared in the irrelevant object $(623 \mathrm{msec})$ or in the background (627 $\mathrm{msec})$. A significant main effect of congruency indicated a Stroop effect $[F(1,30)=$ $\left.225.84, p<.001, \eta_{\mathrm{p}}^{2}=.88\right]$; RTs were shorter in congruent than in incongruent conditions (600 vs. $672 \mathrm{msec}$ ).

The two-way interaction between object similarity and word location was significant $[F(2,29)=4.47, p<.05$, $\left.\eta_{\mathrm{p}}^{2}=.24\right]$, suggesting that words in the relevant object prolonged RTs more strongly with similar objects than with different objects. The two-way interaction between object similarity and congruency was not significant $(F<1)$; however, the two-way interaction between word location and congruency was significant $[F(2,29)=47.62, p<$ $\left..001, \eta_{\mathrm{p}}^{2}=.77\right]$, replicating the previous finding that color words that were in the relevant object produced larger Stroop effects $(D=116 \mathrm{msec})$ than did words that were in the irrelevant object $(D=45 \mathrm{msec})$ or words that were in the background $(D=56 \mathrm{msec})$.

The most important result was a significant three-way interaction indicating that object similarity modulated the two-way interaction between word location and congruency $\left[F(2,29)=7.35, p<.01, \eta_{\mathrm{p}}^{2}=.34\right]$ (see Figure 2). To directly test our prediction that object similarity would modulate the difference between Stroop effects in the irrelevant-object and the background conditions, we conducted a $2 \times 2$ MANOVA, with Stroop effects as the dependent variable. A significant interaction $[F(1,30)=$ $\left.8.21, p<.01, \eta_{\mathrm{p}}^{2}=.22\right]$ showed that Stroop effects from words that were in the irrelevant object $(42 \mathrm{msec})$ were significantly smaller than Stroop effects from words that were in the background $(62 \mathrm{msec})$ when the objects were different, but not when they were similar $(48 \mathrm{msec}$ for words that were in the irrelevant object, and $50 \mathrm{msec}$ for words that were in the background).

\section{Errors}

Error percentages were also fed into a $2 \times 3 \times 2$ MANOVA, and the observed main effects paralleled those of the RT analysis. The significant main effect of word location $\left[F(1,30)=5.01, p<.05, \eta_{\mathrm{p}}^{2}=.14\right]$ indicated more errors with words that were in the relevant object $(5.5 \%)$ than with words that were in the irrelevant object $(1.8 \%)$ or in the background $(2.3 \%)$. The significant main effect of congruency reflected a Stroop effect [congruent condition, $0.3 \%$; incongruent condition, $6.5 \% ; F(1,30)=$ $\left.78.10, p<.001, \eta_{\mathrm{p}}^{2}=.72\right]$. A significant word location $\times$ congruency interaction $\left[F(1,30)=5.68, p<.05, \eta_{\mathrm{p}}^{2}=\right.$ $.16]$ revealed larger Stroop effects from words that were in 


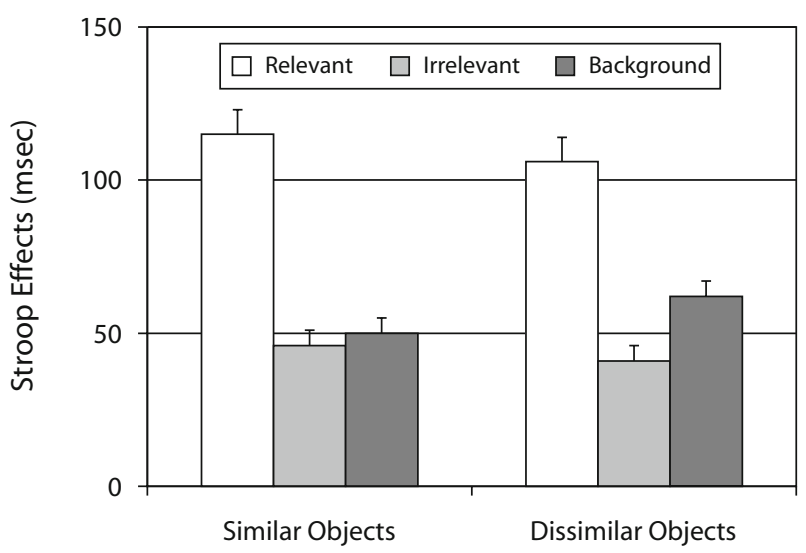

Figure 2. Stroop effects are shown as a function of object similarity (similar vs. dissimilar) and location of the color word (relevant object, irrelevant object, background). Error bars represent the standard errors of the means.

the relevant object $(10.9 \%)$ than from words that were in the irrelevant object $(3.3 \%)$ or in the background (4.6\%). Unlike in the RT analysis, the three-way interaction failed to reach significance in the error analysis $(F<1)$.

\section{DISCUSSION}

The present study investigated the conditions for attentional amplification in the processing of relevant objects and for attentional inhibition in the processing of irrelevant objects. Participants reported the color of one of two superimposed objects that was specified by occlusion. Irrelevant color words were presented as part of the relevant object, as part of the irrelevant object, or in the background. The words were either congruent or incongruent with the color of the relevant object (i.e., with the correct response), and the size of the congruency effects provided a measure of the strength of the processing of the objects relative to each other and to the background. The most interesting manipulation concerned the physical similarity of the relevant and the irrelevant objects: The irrelevant object had the same shape as the relevant object in one session, whereas the irrelevant object had a different shape in the other session.

In both sessions, we observed larger Stroop effects from words belonging to the relevant object than from words that were in the background, indicating that amplification of the relevant object can occur regardless of the similarity between the relevant and the irrelevant objects. In contrast, we observed smaller Stroop effects from words belonging to the irrelevant object than from words that were in the background only when the two objects differed in shape, indicating that this shape difference is both a necessary and a sufficient condition for inhibiting the processing of an irrelevant visual object.

Before addressing the theoretical implications of our findings, we must discuss a methodological peculiarity of our task. As can be seen in Figure 1, words that were inside the objects and words that were outside the objects were presented at different locations. In particular, words that were inside the objects occurred on the horizontal or vertical meridian, whereas words that were outside the objects occurred on a diagonal. We do not believe that the different word locations affected the present pattern of findings, however. First, different word locations cannot explain why words that were inside an object can have both larger effects (when belonging to the relevant object) and smaller effects (i.e., when belonging to the irrelevant object) than do words in the background. Second, different word locations cannot explain the critical three-way interaction (i.e., the dependence of inhibition on object similarity), because the same word locations were used in both sessions. Finally, control experiments that were reported in Wühr and Frings (2008) showed that the exact spatial location of the words inside and outside the objects did not affect the pattern of results, indicating that spatial attention shifts do not play a big role in the present type of task.

The results of the present study have produced converging evidence for a dual-mechanism account of objectbased visual attention. In particular, the results have produced additional evidence for an inhibitory mechanism of object-based attention (see, e.g., Keele \& Neill, 1978; Tipper, 1985). Consistent with the results of Wühr and Frings (2008), we observed that words belonging to the irrelevant object produced smaller Stroop effects than did words that were in the background, despite several methodological differences between the two studies: First, whereas the two shapes were presented at adjacent locations in the former study, the two shapes were both presented at screen center in the present task, minimizing the role of spatial attention shifts toward the relevant object; second, whereas participants selected on the basis of shape in the Wühr and Frings study, participants selected on the basis of a spatial cue (i.e., occlusion) in the present study. We can thus conclude that inhibition of the irrelevant object is a robust phenomenon. Most notably, our paradigm does not suffer from the problems of the NP paradigm that render the interpretation of priming effects equivocal. In the NP paradigm, one measures the effects of ignoring an irrelevant object (in the prime trial) on subsequent processing of a relevant object (in the probe trial), rendering priming effects open to alternative explanations in terms of memory retrieval in the probe trial. In the present paradigm, however, we measure the effects of ignoring an irrelevant object on concurrent processing of a relevant object, excluding alternative explanations referring to memory retrieval or feature overlap between subsequent displays. In order to affect behavior, therefore, amplification and inhibition of stimulus processing must occur before response selection and execution in our paradigm; yet we do not make any claims about the temporal relationship between amplification and inhibition.

Furthermore, the results of the present study provide direct evidence that inhibiting the processing of an irrelevant object requires that this object differ in shape from the relevant object. In contrast, amplifying the processing of a relevant object does not have this requirement. This dissociation is interesting for at least two reasons. First, the fact that attentional inhibition requires more conditions to be 
met than are required by attentional amplification provides a reason why obtaining evidence for attentional inhibition is more difficult than obtaining evidence for attentional amplification. Second, the dissociation suggests that attentional amplification and attentional inhibition work in different ways and possibly operate on different representations. In particular, attentional inhibition may operate on conceptual representations of object shape. This is possible only when the irrelevant object has a different shape than the relevant object, because otherwise inhibition would affect the processing of the relevant object as well.

In summary, the present results provide further support for a dual-process theory of object-based attentional selection. According to this theory, attention amplifies the processing of relevant objects and inhibits the processing of irrelevant objects. The present study demonstrated that, when one selects between two objects, object similarity affects inhibition of the irrelevant object but not amplification of the relevant object; thus, attentional amplification and attentional inhibition work differently or work on different representations.

\section{AUTHOR NOTE}

We thank Julia Bauer and Inga Reismann for collecting the data. Correspondence concerning this article should be addressed to P. Wühr, Institut für Psychologie, Technische Universität Dortmund, EmilFigge-Strasse 50, 44227 Dortmund, Germany (e-mail: wuehr@fk14.tu -dortmund.de).

\section{REFERENCES}

Duncan, J. (1984). Selective attention and the organization of visual information. Journal of Experimental Psychology: General, 113, 501517. doi:10.1037/0096-3445.113.4.501

Fox, E. (1995). Negative priming from ignored distractors in visual selection: A review. Psychonomic Bulletin \& Review, 2, 145-173.

FRINGS, C., \& WüHR, P. (2007). Prime display offset modulates negative priming only for easy-selection tasks. Memory \& Cognition, 35, 504-513.
Houghton, G., \& Tipper, S. P. (1994). A model of inhibitory mechanisms in selective attention. In D. Dagenbach \& T. H. Carr (Eds.), Inhibitory processes in attention, memory, and language (pp. 53-112). San Diego: Academic Press.

Kahneman, D., \& HeniK, A. (1981). Perceptual organization and attention. In M. Kubovy \& J. R. Pomerantz (Eds.), Perceptual organization (pp. 181-211). Hillsdale, NJ: Erlbaum.

Keele, S. W., \& Neill, W. T. (1978). Mechanisms of attention. In E. C. Carterette \& M. P. Friedman (Eds.), Handbook of perception: Perceptual processing (pp. 3-47). New York: Academic Press.

Milliken, B., Joordens, S., Merikle, P. M., \& Seiffert, A. E. (1998). Selective attention: A reevaluation of the implications of negative priming. Psychological Review, 105, 203-229. doi:10.1037/0033 $-295 X .105 .2 .203$

NeiLl, W. T., \& Mathis, K. M. (1998). Transfer-inappropriate processing: Negative priming and related phenomena. In D. L. Medin (Ed.), The psychology of learning and motivation (Vol. 38, pp. 1-44). San Diego: Academic Press.

Rothermund, K., Wentura, D., \& De Houwer, J. (2005). Retrieval of incidental stimulus-response associations as a source of negative priming. Journal of Experimental Psychology: Learning, Memory, \& Cognition, 31, 482-495. doi:10.1037/0278-7393.31.3.482

TIPPER, S. P. (1985). The negative priming effect: Inhibitory priming by ignored objects. Quarterly Journal of Experimental Psychology, 37A, 571-590.

TipPer, S. P. (2001). Does negative priming reflect inhibitory mechanisms? A review and integration of conflicting views. Quarterly Journal of Experimental Psychology, 54A, 321-343. doi:10.1080/ 02724980042000183

WÜHR, P. (2007). A Stroop effect for spatial orientation. Journal of General Psychology, 134, 285-294. doi:10.3200/GENP.134.3.285-294

WÜHR, P., \& FRINGS, C. (2008). A case for inhibition: Visual attention suppresses the processing of irrelevant objects. Journal of Experimental Psychology: General, 137, 116-130. doi:10.1037/0096 $-3445.137 .1 .116$

WÜHR, P., \& WASZAK, F. (2003). Object-based attentional selection can modulate the Stroop effect. Memory \& Cognition, 31, 983-994.

WÜHR, P., \& WeLtLE, M. (2005). Depth cues do not underlie attentional modulations of the Stroop effect. Memory \& Cognition, 33, 676-680.

(Manuscript received September 3, 2008; revision accepted for publication April 8, 2009.) 